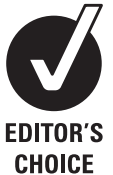

CHOICE

\section{See Editorial, p494 \\ ${ }^{1}$ Department of Cardiovascular Medicine, Tokyo Medical and Dental University, Bunkyo, \\ Tokyo, Japan \\ 2Department of Advanced \\ Clinical Science and \\ Therapeutics, University of \\ Tokyo, Bunkyo, Tokyo, Japan \\ ${ }^{3}$ NB Health Laboratory, \\ Kawaguchi, Saitama, Japan \\ ${ }^{4}$ Department of Cardiovascular \\ Medicine, University of Tokyo, \\ Bunkyo, Tokyo, Japan}

Correspondence to

Professor Jun-ichi Suzuki,

Department of Advanced Clinical

Science and Therapeutics,

University of Tokyo, 7-3-1

Hongo, Bunkyo, Tokyo

113-8655, Japan;

junichisuzuki-circ@umin.ac.jp

Accepted 10 December 2009

\title{
Early treatment with clarithromycin attenuates rat autoimmune myocarditis via inhibition of matrix metalloproteinase activity
}

\author{
Keiichi Hishikari, ${ }^{1}$ Ryo Watanabe, ${ }^{2}$ Masahito Ogawa, ${ }^{2}$ Jun-ichi Suzuki, ${ }^{2}$ \\ Mayumi Masumura, ${ }^{1}$ Tomoko Shimizu, ${ }^{3}$ Kiyoshi Takayama, ${ }^{3}$ Yasunobu Hirata, ${ }^{2}$ \\ Ryozo Nagai, ${ }^{4}$ Mitsuaki Isobe ${ }^{1}$
}

\begin{abstract}
Background Matrix metalloproteinase (MMP) activity is upregulated in the hearts with myocarditis, and its activation contributes to the changes in left ventricular function. A major macrolide antibiotic, clarithromycin (CAM), has many biological functions including MMP regulation. However, little is known about the effect of CAM in myocarditis via MMPs.
\end{abstract}

Objective To clarify the role of MMPs regulated by CAM in the progression of myocarditis.

Design CAM was given to experimental rats with autoimmune myocarditis (EAM) from day -7 to day 21 (early treated group, $n=6$ ) or from day 1 to day 21 (late treated group, $n=6$ ) twice a day.

Results Although the non-treated rats showed blood pressure decline and impaired cardiac function, early CAM treatment prevented this progression. Pathologically, severe myocardial cell infiltration $(30.5 \pm 4.2 \%)$ and fibrosis $(32.2 \pm 1.1 \%)$ were detected in the non-treated group, while early CAM treatment significantly suppressed these changes (infiltration $6.5 \pm 0.2 \%$, fibrosis $5.9 \pm 3.9 \%)$. Zymography showed that non-treated EAM resulted in enhanced ventricular activities of MMP-9, while early CAM treatment reduced the alteration. However, late CAM treatment was less effective than the early treatment.

Conclusions Early CAM treatment is effective to attenuate myocarditis by suppressing MMP-9.

\section{INTRODUCTION}

Myocarditis is an inflammatory heart disease which is considered as one of the main causes of dilated cardiomyopathy. Although viral infection is the most common cause of human myocarditis, autoimmune myocarditis also occurs as a giant cell myocarditis. ${ }^{1} 2$ A novel model of rat experimental autoimmune myocarditis (EAM) has been used to investigate the pathogenesis of myocarditis induced by autoimmune mechanisms. ${ }^{3}$ Because matrix metalloproteinases (MMPs) have a crucial role in the recruitment of inflammatory cells and remodelling of the inflamed myocardium, ${ }^{3}{ }^{4}$ we focused our research on the roles of MMPs in the development of myocarditis.

Inflammatory cells such as lymphocytes ${ }^{5}$ and monocytes/macrophages ${ }^{6}$ produce MMPs, and levels have been shown to be upregulated in inflamed hearts. ${ }^{7}$ MMPs are a large family of proteinases that proteolytically degrade extracellular matrix. The degradation of extracellular matrix is an important event in the process of inflammation and tissue remodelling. A member of the MMPs, MMP-9 (gelatinase B), has an important role in tissue remodelling and the migration of smooth muscle cells, macrophages and other cells. ${ }^{8}$

Clarithromycin (CAM) is a 14 -member ring macrolide and a potent antibiotic for the treatment of various microbial infections. CAM has been reported to have multiple biological effects, such as alteration of inflammatory factors and MMPs. ${ }^{9}{ }^{10}$ Although we have shown that CAM suppresses cardiac rejection using murine heart transplant models, ${ }^{11}$ little is known about the effects of CAM in myocarditis.

This study, for the first time, demonstrates that CAM suppresses acute myocarditis through the inhibition of MMP-9 expression and activity.

\section{MATERIALS AND METHODS \\ Reagents and animals}

CAM was kindly provided by Taisho Toyama Pharmaceutical (Tokyo, Japan). Male Lewis rats (6 weeks old, 200-250 g) were purchased from Sankyo Laboratories (Tokyo, Japan). Rats were given CAM (100 mg/kg/day) orally twice a day. We selected the dose for rats based on our previous paper. ${ }^{11}$ Rats with EAM were divided into three groups: (a) early treated group (from day -7 to day $21, \mathrm{n}=6) ;(b)$ late treated group (from day 1 to day $21, \mathrm{n}=6$ ) or $(c)$ vehicle (untreated group, $\mathrm{n}=6$ ) for control. They were maintained in compliance with the animal welfare guidelines of the Institute of Experimental Animal, Tokyo Medical and Dental University. Our investigation also conformed with the Guide for the Care and Use of Laboratory Animals published by the US National Institutes of Health (NIH Publication No 85-23, revised 1996).

\section{Induction of EAM}

Purified porcine cardiac myosin (Sigma, St Louis, Missouri, USA, lot 063K7013) was emulsified with an equal volume of adjuvant complete Freund (Difco (BD Diagnostic), Franklin Lakes, New Jersey, USA) supplemented with Mycobacterium tuberculosis (Difco, lot 5136544) to a final concentration of $5 \mathrm{mg} / \mathrm{ml}$. Rats were injected subcutaneously in each footpad with $0.1 \mathrm{ml}$ emulsion $(0.2 \mathrm{ml} / \mathrm{rat})$. The day of myosin injection was defined as day $0 .{ }^{12}$

\section{Haemodynamic measurement}

Blood pressure was measured on days 0, 14, 19, 21 in all rats in a conscious state by using a tail-cuff system (BP-98A; Softron, Tokyo, Japan). 


\section{Echocardiogram}

A transthoracic echocardiogram was obtained on days 0,14 and 21 under anaesthesia (pentobarbital sodium $5.0 \mathrm{mg} / 100 \mathrm{~g}$ ). The M-mode echocardiograph was performed at the papillary muscle level, and the left ventricular ejection fraction was calculated as described before ${ }^{13} 14$ using an echocardiographic machine with a 7.5 MHz transducer (Nemio, Toshiba, Tokyo, Japan).

\section{Histological examination}

The heart sections were stained by haematoxylin and eosin $(\mathrm{H} \& \mathrm{E})$ or the Mallory method. The areas of the entire hearts and regions affected by myocarditis were determined by a computerassisted analyser (Scion Image beta 4.02; Scion Corporation, Frederick, Maryland, USA). The area ratio was calculated as described previously. ${ }^{11} 13$

\section{Immunohistochemistry}

Frozen sections were incubated with anti-rat CD4 (OX-35), CD8 (OX-8) (Pharmingen, San Diego, California, USA), MMP-2 (H-76), MMP-9 (H-129) (Santa Cruz Biotechnology, Santa Cruz, California, USA) or CD68 (ED1, AbD serotec, Oxford, UK) antibody (each at $1-10 \mu \mathrm{g} / \mathrm{ml}$ ) for $8 \mathrm{~h}$ at $4^{\circ} \mathrm{C}$. Histofine simple stain rat MAX-PO (MULTI) was used as a secondary antibody. ${ }^{11} 13$

\section{Film in situ zymography}

Frozen sections were mounted on polyethylene telephthalate film coated with gelatin (FIZ-GN) (Wako Pure Chemical Industries, Osaka, Japan). After incubation, these films were dried for $30 \mathrm{~min}$, and then stained with Biebrich Scarlet Stain Solution (Wako) for $4 \mathrm{~min}$. Gelatinolytic activity is visible as a clear area unstained by Biebrich Scarlet. ${ }^{15}$

\section{Real-time RT-PCR}

Total RNA was isolated from the hearts and cDNA was prepared with the RT-PCR kit (Stratagene, La Jolla, California, USA). Real-time PCR in a StepOne real-time PCR system (Applied Biosystems, ABI, Carlsblad, California, USA) was used to determine the mRNA expression of tumour necrosis factor $\alpha$ (assay ID: Rn99999017_m1), interleukin 10 (assay ID: Rn00563409_m1), CCL2 (assay ID: Rn00580555_m1) and 18s rRNA (assay ID: Hs99999901_s1) as a control. The real-time PCR protocol consisted of an initial step at $95^{\circ} \mathrm{C}$ for $20 \mathrm{~s}$, followed by 50 cycles: $95^{\circ} \mathrm{C}$ for $1 \mathrm{~s}$, annealing at $60^{\circ} \mathrm{C}$ for $20 \mathrm{~s}$. cDNA was run in duplicate and quantitative data were calculated using the comparative $\mathrm{C}_{\mathrm{T}}\left(\Delta \Delta \mathrm{C}_{\mathrm{T}}\right)$ method.

\section{Statistical analysis}

Values are given as mean \pm SEM. Groups were compared with Scheffé's ANOVA. We used Student t test for comparisons between two groups. Differences were considered statistically significant at a value of $\mathrm{p}<0.05$.

\section{RESULTS}

\section{Blood pressure and heart rates}

Early CAM treatment reduced the myocarditis-induced decrease in mean blood pressure compared with the untreated group on days 19 and 21. Late CAM treatment did not negate the blood pressure decline (figure 1). Heart rates were not different among the groups.

\section{CAM improved cardiac function}

Early CAM treatment prevented impairment of left ventricular contraction on day 21 . The improvements in cardiac wall movements together with the lesser pericardial effusion were observed in the early CAM-treated group. However, late CAM treatment had fewer effects on contraction and pericardial effusion (figure 1).

\section{CAM reduced heart per body weight ratios}

Non-treated EAM hearts demonstrated an increased heart per body weight ratio $(0.55 \pm 0.05 \%)$ compared with that of native rats $(0.30 \pm 0.10 \%, p<0.05)$. The early CAM administration significantly reduced the ratio $(0.35 \pm 0.01 \%, \mathrm{p}<0.05)$ compared with that of the non-treated EAM hearts. However, late CAM treatment had less effect on heart per body weight $(0.51 \pm 0.05 \%)$

\section{Reduction of the myocarditis-affected areas by CAM}

On day 21, severe myocardial cell infiltration and fibrosis were seen in the non-treated hearts. However, the affected lesions
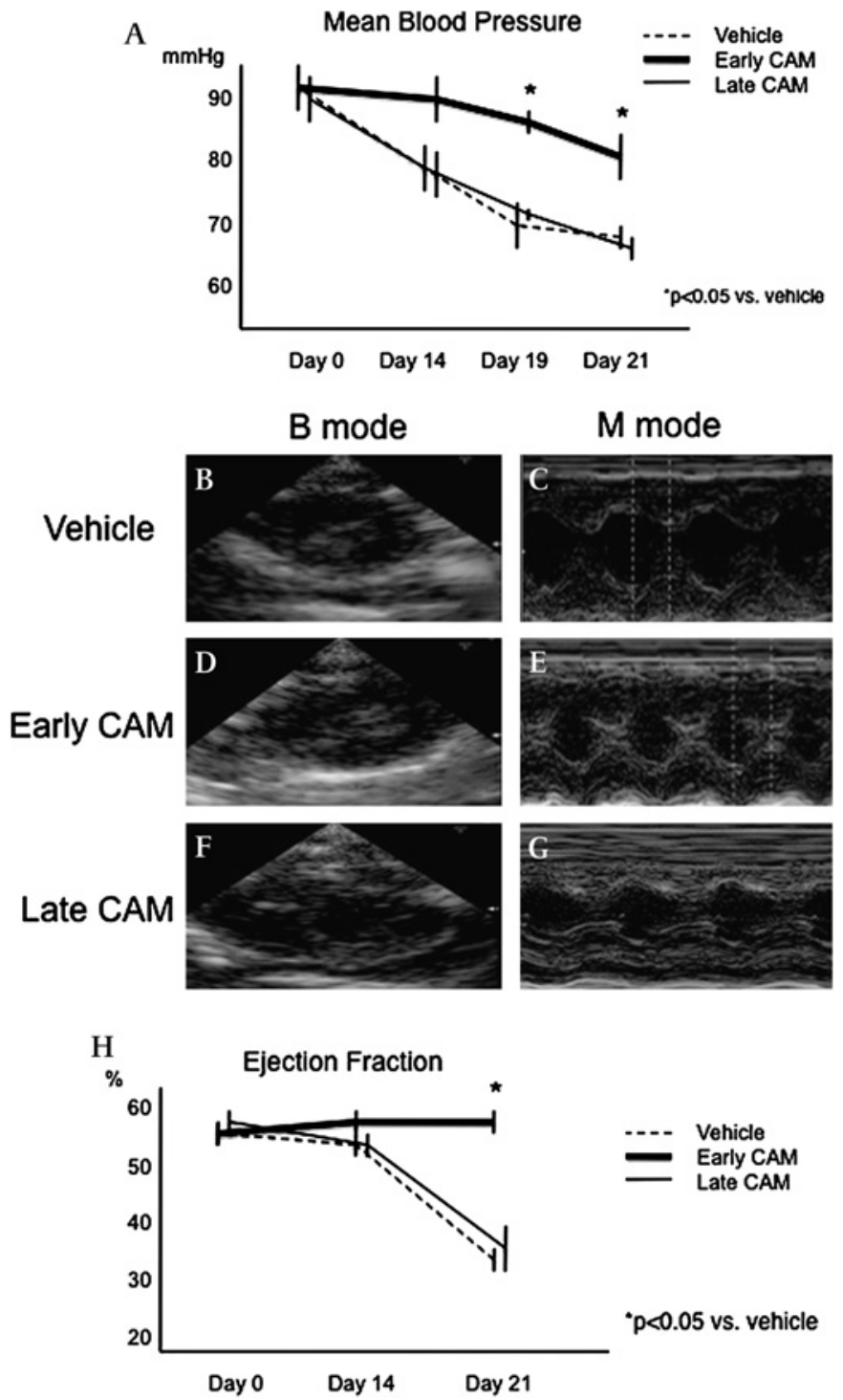

Figure 1 Blood pressure and echocardiogram. (A) Mean blood pressure. Early clarithromycin (CAM) reduced the myocarditis-induced decrease in mean blood pressure compared with the untreated group on days 19 and 21. Late CAM treatment did not negate the blood pressure decline. $(\mathrm{B}-\mathrm{H})$ Echocardiograms. The improvements in cardiac wall movements together with the lesser pericardial effusion $(D, E)$ were seen in the early CAM-treated group compared with those in the untreated group $(B, C)$. However, late CAM treatment had fewer effects on contraction and pericardial effusion $(F, G)$. $(H)$ Ejection fraction greatly improved in the early treated group in comparison with the untreated group on day $21 .{ }^{*} \mathrm{p}<0.05$ versus vehicle. 
significantly decreased in the hearts treated with early CAM treatment. Late CAM treatment showed no statistical improvement in affected and fibrosis areas (figure 2).

\section{Immunohistochemical findings}

We detected the expression of CD4, CD8, ED-1, MMP-2 and MMP-9 positive infiltrating cells in the untreated group on day 21. All these positive cells were fewer in the early CAM-treated group than in the untreated group (figure 3 ).

\section{MMP inhibition}

Activity of MMPs was markedly enhanced in the infiltrated area in the non-treated group; however, early CAM-treated hearts attenuated the MMP activity (figure 4).

\section{Transcriptions of inflammatory mediators}

Real-time RT-PCR demonstrated that early CAM treatment did not alter the transcription levels of inflammatory factors, such as tumour necrosis factor $\alpha$, interleukin 10 and CCL2, statistically (figure 4).

\section{DISCUSSION}

CAM is known to be a potent antibiotic for the treatment of various microbial infections but also has multiple biological effects. While the 16-member ring macrolide does not have multiple effects, it is believed that the multiple effect may be
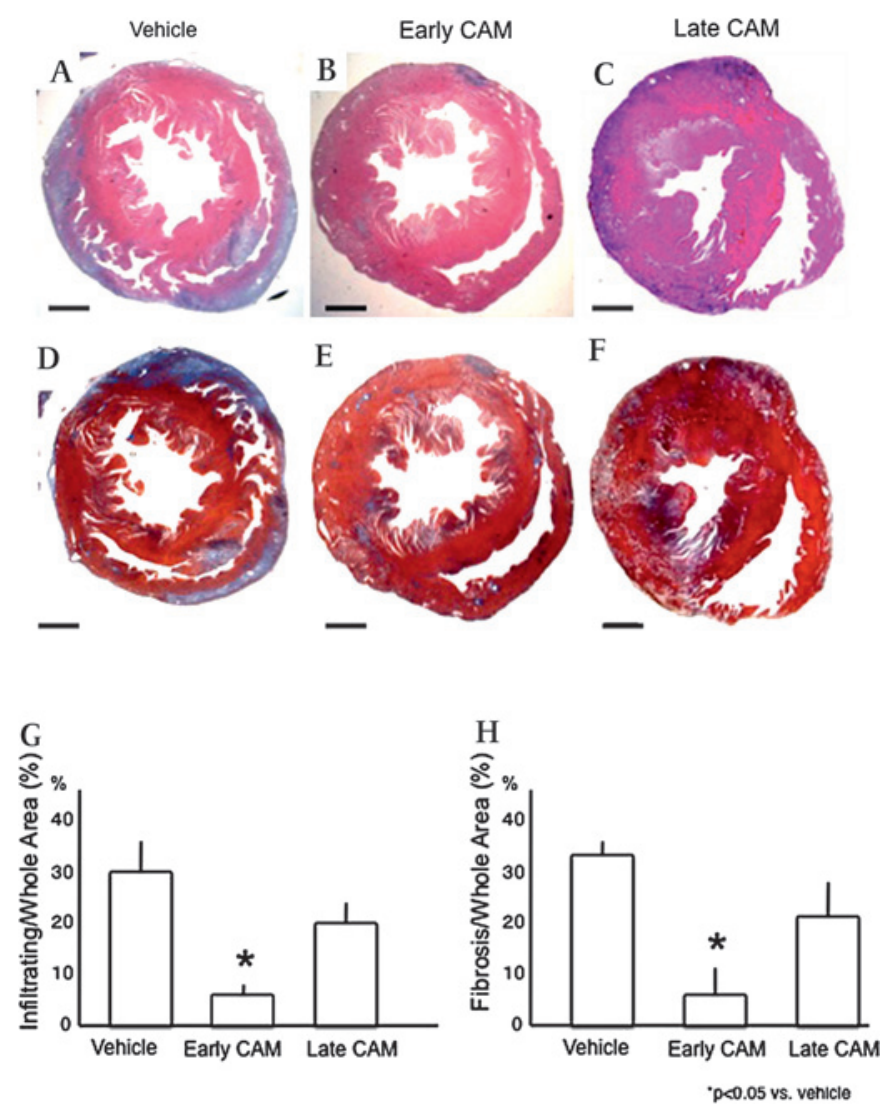

Figure 2 H\&E and Mallory staining. While severe myocardial cell infiltration and fibrosis was seen in the non-treated hearts on day 21 $(A, D)$, myocardial lesions significantly decreased in early clarithromycin (CAM)-treated hearts $(B, E)$. However, late CAM treatment showed no statistical improvement $(\mathrm{C}, \mathrm{F})$. $(\mathrm{G}, \mathrm{H})$ Quantitative data. Scale bars: $2 \mathrm{~mm}$; ${ }^{*} \mathrm{p}<0.05$ versus vehicle. characteristic of 14-member ring macrolides. ${ }^{9}{ }^{10}$ A long-term 14 member ring macrolide clinical treatment has been established for diffuse panbronchiolitis. These drugs are effective via antiinflammatory mechanisms other than antibacterial activity. In this study, we demonstrated that CAM inhibited expression of MMP-9 and suppressed the myocardial inflammation in the rat EAM model.

T lymphocytes and macrophages are a major component of the cellular infiltration of EAM, they contribute to the development of tissue injury in acute inflammatory reaction. ${ }^{16}$ Thus, studies have shown that the conventional immunosuppressive drugs attenuated EAM. ${ }^{17}{ }^{18}$ MMPs have an important role in cell migration and are involved in the migratory capabilities of inflammatory cells such as T cells. ${ }^{19}$ MMP-9 is especially important in inflammation, the gelatinase have been shown to have a critical role in the process of infiltration in inflamed tissues. ${ }^{20}$ Cheung et al examined the severity of coxsackievirus B3-induced myocarditis in MMP-9-deficient mice. They showed that infected MMP-9-deficient mice had greater myocardial injury and foci of
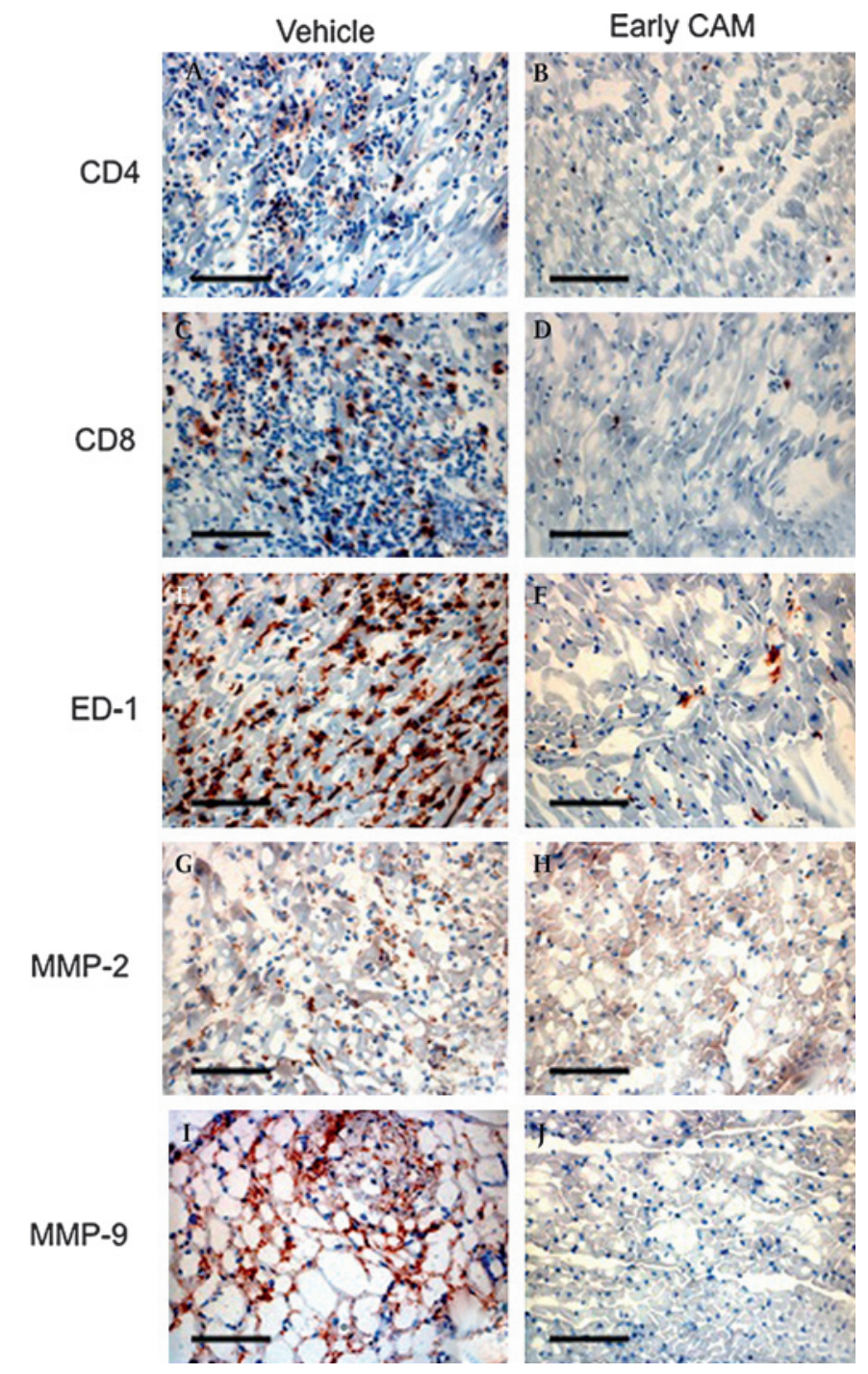

Figure 3 Immunohistochemistry. Enhanced expression was detected of CD4 (A), CD8 (C), ED-1 (E), matrix metalloproteinase (MMP)-2 (G) and MMP-9 (I)-positive cells in the untreated group on day 21. Fewer of all the above-mentioned positive cells were found in the early clarithromycin (CAM)-treated group $(B, D, F, H, J)$ than in the untreated group. Scale bars: $25 \mu \mathrm{m}$. 
Vehicle
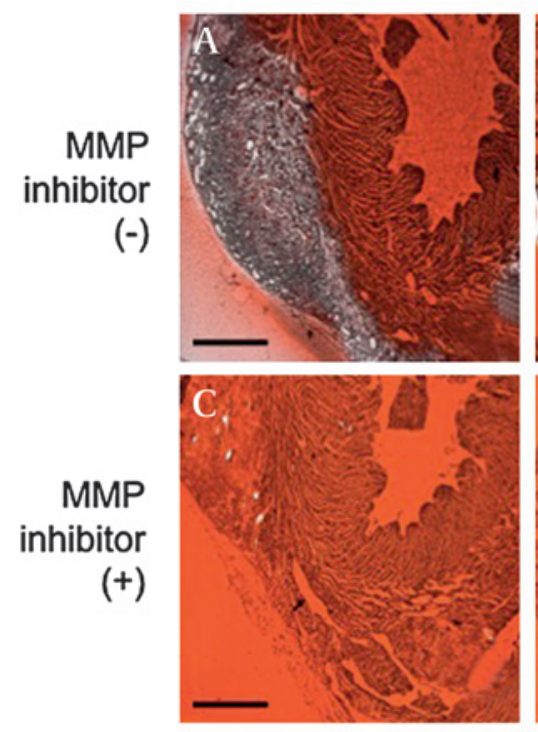

$(+)$

\section{Early CAM}
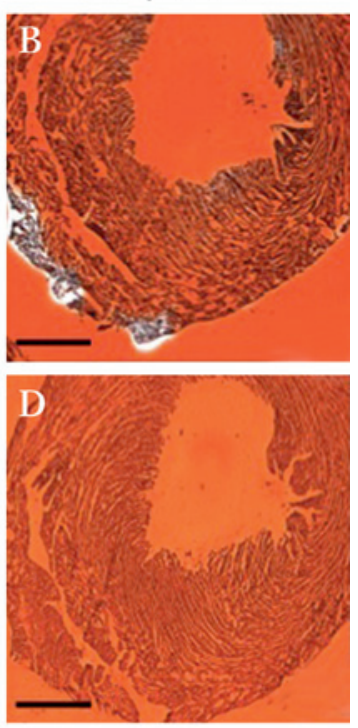

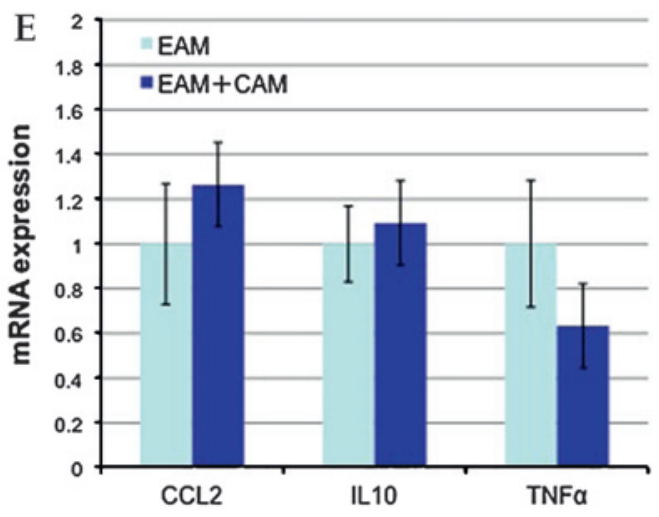

Figure 4 In situ film zymography and real-time RT-PCR. Matrix metalloproteinase (MMP) activity was markedly enhanced in the infiltrated area in the non-treated group $(A)$; however, early clarithromycin (CAM)-treated hearts attenuated the MMP activities (B). MMP inhibitor $(+)$ films $(C, D)$ show the non-gelatinase specific activity. Scale bars: $1 \mathrm{~mm}$. Real-time RT-PCR demonstrated that early CAM treatment did not alter the transcription levels of inflammatory factors, such as tumour necrosis factor $\alpha$ (TNF $\alpha$ ), interleukin (IL)10 and CCL2, statistically (E).

infection than wild-type mice. ${ }^{20}$ The effect of MMP-9 in myocarditis seems to be paradoxical because we showed that MMP-9 inhibition suppressed EAM development. The reason may be the difference between the models (mice vs rats, and viral infection vs autoimmune reaction) and interventions (gene modification vs pharmacological treatment).

It has been reported that CAM inhibits the activation of $\mathrm{NF}-\kappa \mathrm{B}^{11} 22^{22}$; the transcriptional factor is well known as the regulator of the MMP-9. On the other hand, other papers have shown that erythromycin, one of the 14-member ring macrolides, suppresses the production of both MMP-2 and MMP-9 in vitro. $^{23}$ These results demonstrate that CAM suppresses MMP-9 activity; this resulted in attenuated myocardial inflammatory cell infiltration, such as $\mathrm{CD} 4+$ and $\mathrm{CD} 8+$ cells. This alteration consequently decreased the myocardial remodelling compared with the control group. However, the detailed mechanism between CAM and MMP-9 has not yet been elucidated. Further investigation is needed.
As we have shown, late CAM treatment did not effectively suppress EAM compared with early CAM treatment. We reported that catechins showed similar results, while a COX-2 inhibitor had different results using the same rat EAM model. ${ }^{13} 14$ These findings may be caused by a different time course of targeted factor expression. It was shown that MMP activity was already present when cell infiltration began in Cprotein-induced murine autoimmune myocarditis, ${ }^{24}$ and thus early CAM treatment was deemed more effective than late CAM treatment in suppressing EAM development. Thus, CAM administration may be useful for the prevention of recurrence and chronic remodelling after myocarditis. Although inflammatory cytokines partially modulate EAM development, ${ }^{25}$ CAM did not alter the transcription levels in this study, statistically. It has been reported that CAM mainly alters MMP activities, whereas it has relatively fewer effects on cytokines in the cardiac allograft rejection. ${ }^{11}$ Therefore, MMPs have a key role in the development of EAM regulated by CAM.

This study has a limitation. The objective of this study was to clarify the role of MMPs regulated by CAM in the progression of EAM. This aim was not achieved, as the results of this study describe the effects of CAM on inflammatory status and clinical outcome in myocarditis rather than providing an insight into the mechanism. The relative failure (a trend only) of the late CAM treatment may be due to the small numbers of animals tested.

In conclusion, CAM may be used in the suppression of myocardial remodelling after acute myocarditis, cardiovascular and other inflammatory diseases in clinical settings.

Acknowledgements We thank Ms Noriko Tamura and Ms Yasuko Matsuda for their excellent assistance.

Funding This study was supported by grants from a Grant-in-aid from the Japan Society for the Promotion of Science.

Competing interests None.

Patient consent Obtained

Provenance and peer review Not commissioned; not externally peer reviewed.

\section{REFERENCES}

1. Cooper LT Jr. Myocarditis. N Engl J Med 2009;360:1526-38.

2. Kawai C. From myocarditis to cardiomyopathy: mechanism of inflammation and cell death. learning from the past for the future. Circulation 1999;99:1091-100.

3. Kodama M, Hanawa H, Saeki M, et al. Rat dilated cardiomyopathy after autoimmune giant cell myocarditis. Circ Res 1994;75:278-84.

4. Heymans S, Pauschinger M, De Palma A, et al. Inhibition of urokinase-type plasminogen activator or matrix metalloproteinases prevents cardiac injury and dysfunction during viral myocarditis. Circulation 2006;114:565-73.

5. Xia M, Leppert D, Hauser SL, et al. Stimulus specificity of matrix metalloproteinase dependence of human $\mathrm{T}$ cell migration through a model basement membrane. $\mathrm{J}$ Immunol 1996;156:160-7.

6. Hashimoto $\mathbf{N}$, Kawabe $T$, Hara $T$, et al. Effect of erythromycin on matrix metalloproteinase-9 and cell migration. J Lab Clin Med 2001;137:176-83.

7. Li J, Schwimmbeck PL, Tschope C, et al. Collagen degradation in a murine myocarditis model: relevance of matrix metalloproteinase in association with inflammatory induction. Cardiovasc Res 2002;56:235-47.

8. Cho A, Reidy MA. Matrix metalloproteinase- 9 is necessary for the regulation of smooth muscle cell replication and migration after arterial injury. Circ Res 2002;91:845-51.

9. Kanai K, Asano K, Hisamitsu T, et al. Suppression of matrix metalloproteinase-9 production from neutrophils by a macrolide antibiotic, roxithromycin, in vitro. Mediators Inflamm 2004;13:313-19.

10. Kanai K, Asano K, Hisamitsu T, et al. Suppression of matrix metalloproteinase production from nasal fibroblasts by macrolide antibiotics in vitro. Eur Respir $J$ 2004;23:671-8.

11. Ogawa M, Suzuki J, Hishikari K, et al. Clarithromycin attenuates acute and chronic rejection via MMP suppression in murine cardiac transplantation. J Am Coll Cardiol 2008;51:1977-85.

12. Futamatsu H, Suzuki J, Kosuge $\mathrm{H}$, et al. Attenuation of experimental autoimmune myocarditis by blocking activated T cells through inducible costimulatory molecule pathway. Cardiovasc Res 2003;59:95-104. 
13. Suzuki J, Ogawa M, Futamatsu H, et al. A cyclooxygenase-2 inhibitor alters Th1/Th2 cytokine balance and suppresses autoimmune myocarditis in rats. $J \mathrm{Mol}$ Cell Cardiol 2006;40:688-95.

14. Suzuki J, Ogawa M, Futamatsu $\mathrm{H}$, et al. Tea catechins improve left ventricular dysfunction, suppress myocardial inflammation, fibrosis, and alter cytokine expression in rat autoimmune myocarditis. Eur J Heart Fail 2007;9:152-9.

15. Wright JW, Brown TE, Harding JW. Inhibition of hippocampal matrix metalloproteinase-3 and -9 disrupts spatial memory. Neural Plast 2007;2007:73813.

16. Maeda K, Shioi T, Kosugi R, et al. Rapamycin ameliorates experimental autoimmune myocarditis. Int Heart J 2005;46:513-30.

17. Kamiyoshi Y, Takahashi M, Yokoseki O, et al. Mycophenolate mofetil prevents the development of experimental autoimmune myocarditis. J Mol Cell Cardiol 2005;39:467-77.

18. Kodama M, Hanawa $\mathrm{H}$, Zhang $\mathrm{S}$, et al. FK506 therapy of experimental autoimmune myocarditis after onset of the disease. Am Heart J 1993:126:1385-92.

19. Mazzarella G, Petillo O, Margarucci S, et al. Role of monocyte/macrophage population in immune response. Monaldi Arch Chest Dis 1998;53:92-6.
20. Cheung C, Marchant D, Walker EK, et al. Ablation of matrix metalloproteinase-9 increases severity of viral myocarditis in mice. Circulation 2008;117:1574-82.

21. Kikuchi T, Hagiwara K, Honda Y, et al. Clarithromycin suppresses lipopolysaccharideinduced interleukin-8 production by human monocytes through AP-1 and NF-kappa B transcription factors. J Antimicrob Chemother 2002;49:745-55.

22. Abe S, Nakamura $\mathrm{H}$, Inoue $\mathrm{S}$, et al. Interleukin-8 gene repression by clarithromycin is mediated by the activator protein-1 binding site in human bronchial epithelial cells. Am J Respir Cell Mol Biol 2000;22:51-60.

23. Guo $\mathbf{H}$, Lee JD, Yue $\mathrm{H}$, et al. Effect of erythromycin on homocysteine-induced extracellular matrix metalloproteinase-2 production in cultured rat vascular smooth muscle cells. Indian J Med Res 2005;121:764-70.

24. Matsumoto Y, Park IK, Kohyama K. Matrix metalloproteinase (MMP)-9, but not MMP-2, is involved in the development and progression of $C$ protein-induced myocarditis and subsequent dilated cardiomyopathy. J Immunol 2009;183:4773-81.

25. Yokoseki $\mathbf{0}$, Suzuki J, Kitabayashi $\mathrm{H}$, et al. cis element decoy against neculear factor$\mathrm{kB}$ attenuates development of experimental autoimmune myocarditis in rats. Circ Res 2001;89:899-906. 